\title{
Stable commutator length in word-hyperbolic groups
}

\author{
Danny Calegari and Koji Fujiwara
}

\begin{abstract}
In this paper we obtain uniform positive lower bounds on the stable commutator length of elements in word-hyperbolic groups and certain groups acting on hyperbolic spaces (namely the mapping class group acting on the complex of curves, and an amalgamated free product acting on an associated Bass-Serre tree). If $G$ is a word-hyperbolic group that is $\delta$-hyperbolic with respect to a symmetric generating set $S$, then there is a positive constant $C$ depending only on $\delta$ and on $|S|$ such that every element of $G$ either has a power which is conjugate to its inverse, or else the stable commutator length of the element is at least equal to $C$. By Bavard's theorem, these lower bounds on stable commutator length imply the existence of quasimorphisms with uniform control on the defects; however, we show how to construct such quasimorphisms directly.

We also prove various separation theorems on families of elements in such groups, constructing homogeneous quasimorphisms (again with uniform estimates) which are positive on some prescribed element while vanishing on some family of independent elements whose translation lengths are uniformly bounded.

Finally, we prove that the first accumulation point for stable commutator length in a torsionfree word-hyperbolic group is contained between $1 / 12$ and $1 / 2$. This gives a universal sense of what it means for a conjugacy class in a hyperbolic group to have a small stable commutator length, and can be thought of as a kind of "homological Margulis lemma".
\end{abstract}

Mathematics Subject Classification (2010). 20F65, 20F32, 57M07.

Keywords. Quasimorphism, stable commutator length, scl, hyperbolic group, pleated surface, Mineyev's flow space, mapping class group, defect.

\section{Introduction}

Let $G$ be a group, and let $[G, G]$ denote the commutator subgroup. Given $g \in[G, G]$ the commutator length of $g$, denoted $\operatorname{cl}(g)$, is the least number of commutators in $G$ whose product is equal to $g$. The stable commutator length, denoted $\operatorname{scl}(g)$, is the limit of $\operatorname{cl}\left(g^{n}\right) / n$ as $n$ goes to infinity. If $g^{n} \in[G, G]$ for some least positive integer $n$, define $\operatorname{scl}(g)=\operatorname{scl}\left(g^{n}\right) / n$, and define $\operatorname{scl}(g)=\infty$ if no power of $g$ is contained in $[G, G]$; see $\S 2$ for precise definitions. For a general introduction to the theory of stable commutator length, see [6]. 
Informally, if $G=\pi_{1}(X)$ for some topological space $X$, commutator length is the smallest genus of a surface in $X$ whose boundary represents a given loop in $X$, and stable commutator length "rationally" measures the same quantity. At the homological level, stable commutator length is an $L^{1}$ filling norm with $\mathbb{Q}$ coefficients, in the sense of Gersten [18] or Gromov [20].

This paper is concerned with obtaining uniform positive lower bounds on stable commutator length in (word-) hyperbolic groups and groups acting on $(\delta$-) hyperbolic spaces. Morally, $(\delta$-) hyperbolic spaces are those whose geometry can be efficiently probed by maps of triangles and other surfaces into the space, so it should not be surprising that they can be studied effectively with stable commutator length. However, there is a sense in which our results are counterintuitive, which we briefly explain.

It is a widely observed fact that in the presence of (coarse) negative curvature, one can obtain upper bounds on the "size" of a surface which is efficient in some sense. Here size is measured with respect to some kind of norm; for instance area, Whitney b-norm, $L^{1}$ norm on homology etc. Efficiency might vary from context to context (e.g. harmonic, minimal, normal) and will depend on the way in which we measure the size of the surface. Our main results say that in the presence of (coarse) negative curvature, there is a uniform (positive) lower bound on the (homological) size of certain surfaces.

We concentrate on worst-case behavior, rather than typical behavior, and our results are frequently sharp. Other authors have studied commutator length and its relation with negative curvature, especially Gromov [22], §6.C. $C_{2}$. In our language, Gromov observes that in a word-hyperbolic group, if $g \in[G, G]$ is not torsion and does not have a power conjugate to its inverse, then $\operatorname{scl}(g)>0$ (actually, Gromov neglects to mention the second possibility). The key innovation in our paper is that our bounds are uniform, and depend only on macroscopic features of the group, specifically $\delta$ and number of generators. Note that any hyperbolic group may be made $\delta$-hyperbolic for some universally small $\delta$ (say $\delta<10$ ) just by increasing the number of generators, so our results in this sense are best possible.

Perhaps our most striking theorem is Theorem B, a kind of "spectral gap" theorem, which says that in a non-elementary torsion-free word-hyperbolic group, the first accumulation point for stable commutator length as a function from conjugacy classes to positive real numbers is between $1 / 12$ and $1 / 2$. These bounds imply that there is a universal sense of what it means for an element in a word-hyperbolic group to have a small stable commutator length. This should perhaps be compared with Margulis' Lemma, which says that there is a universal sense of what it means for a closed geodesic in a hyperbolic manifold to be short. The difficulty in transporting Margulis' Lemma to geometric group theory is that geometric notions in a group are typically only defined up to a certain ambiguity (e.g. quasi-isometry) which obscures small scale geometric phenomena. The advantage of working with stable commutator length, and the power of our theorem, is precisely that it captures such small scale phenomena. This comparison is more than superficial - the key to obtaining the sharp lower bound is to use estimates due to Mineyev which reproduce, in a gen- 
eral $\delta$-hyperbolic space, geometric phenomena which are strictly analogous to $C^{1}$ phenomena in Lie groups.

We are able to give similar uniform lower bounds on the stable commutator length of certain elements in two other important classes of groups: mapping class groups of surfaces (hereafter denoted $\operatorname{MCG}(S)$ ), and amalgamated free products. In general, neither kind of group is $\delta$-hyperbolic, but each acts naturally on a certain $\delta$-hyperbolic space: the complex of curves, and the Bass-Serre tree respectively. Obtaining lower bounds on stable commutator length in mapping class groups is intimately tied to important problems in 4-dimensional symplectic geometry; for example, estimating the complexity of a symplectic 4-manifold filling a given contact 3-manifold, or controlling the ratios of characteristic numbers (e.g. Euler characteristic and signature). This point of view has been pioneered by D. Kotschick, sometimes in collaboration with H. Endo, in a series of papers which include [25], [12], [26] and [13]. From another point of view, there are relations between symplectic geometry and stable commutator length which are more intimately connected with invariants like Hofer length and subgroup distortion; see work of Polterovich, especially [33].

The Bavard Duality Theorem (see Theorem 2.5) gives a duality between stable commutator length, and certain kinds of functions on a group, called homogeneous quasimorphisms. A function $f: G \rightarrow \mathbb{R}$ is a homogeneous quasimorphism if it is homogeneous (i.e. if it satisfies $f\left(g^{n}\right)=n f(g)$ for all $g \in G$ and $n \in \mathbb{Z}$ ) and if there is a least real number $D(f)$ called the defect, for which $|f(g h)-f(g)-f(h)| \leq$ $D(f)$ for all $g, h \in G$. The Duality Theorem says that obtaining lower bounds for stable commutator length is equivalent to constructing homogeneous quasimorphisms with small defects (see $\S 2$ ). Bavard's theorem is non-constructive, and uses the Axiom of Choice (in the form of the Hahn-Banach Theorem); however in our paper we are able to construct explicit quasimorphisms with small defects directly. We are also able to prove various separation theorems, constructing quasimorphisms with small defects that take big values on prescribed elements and vanish on others. This is a quantitative improvement on the kinds of separation theorems proved or conjectured by various people in the past, and we expect it to have a number of applications.

Let us stress that this paper is concerned more with developing foundations, and understanding what (to us) seems like a fundamental algebraic/geometric inequality, manifested in several important group theoretic contexts, than with deducing topological or other corollaries.

1.1. Statement of results. We now give a brief summary of the contents of the paper. In $\S 2$ we give definitions, standardize notation, and recall some basic elements of the theory of $\delta$-hyperbolic groups and spaces, and bounded cohomology. We spend some time discussing Mineyev's geodesic flow space, which is a technical tool that we find useful for establishing uniform bounds on stable commutator length directly by means of geometry rather than using quasimorphisms (see $\S 2$ for definitions).

There are other versions of the geodesic flow space of a word-hyperbolic group or space, and that of Gromov [21] is probably best known. However, Mineyev's space 
has some features that make it technically easier to use for our purposes.

In $\S 3$ we give the first version of our first main result, the Gap Theorem:

Theorem A (Gap Theorem, weak version). Let $G$ be a word-hyperbolic group that is $\delta$-hyperbolic with respect to a symmetric generating set $S$ with $|S|$ generators. Then there is a constant $C(\delta,|S|)>0$ such that for every $a \in G$, either $\operatorname{scl}(a) \geq C$ or else there is some positive integer $n$ and some $b \in G$ such that $b a^{-n} b^{-1}=a^{n}$.

Note that if $b a^{-n} b^{-1}=a^{n}$ then $\operatorname{scl}(a)=0$.

It is easy to produce examples of word-hyperbolic groups which contain elements with arbitrarily small (positive) stable commutator length, so the dependence on $\delta$ and $|S|$ is necessary.

In $\S 4$ and $\S 5$ we introduce so-called counting quasimorphisms, and use them to prove the second (stronger) version of the Gap Theorem. In the following theorem, we use the notation $\tau(a)$ to denote the translation length of an element $a \in G$, as measured in the Cayley graph $\Gamma_{S}(G)$ of $G$ with respect to a fixed generating set $S$. It is worth keeping in mind that if $a$ is not torsion, there is a positive lower bound on $\tau(a)$ that depends only on $\delta$ and $|S|$.

Theorem A' (Gap Theorem, strong version). Let $G$ be a word-hyperbolic group that is $\delta$-hyperbolic with respect to a symmetric generating set $S$ with $|S|$ generators. Let $a \in G$ be a (non-torsion) element. Assume that there is no $n>0$ and no $b \in G$ with $b a^{-n} b^{-1}=a^{n}$. Then there is a homogeneous quasimorphism $h$ on $G$ such that

(1) $h(a)=1$,

(2) the defect of $h$ is $\leq C(\delta,|S|)$.

Moreover, let $a_{i} \in G$ be a collection of elements for which $T=\sup _{i} \tau\left(a_{i}\right)$ is finite. Suppose that for all integers $n, m \neq 0$ and all elements $b \in G$ and indices $i$ there is an inequality

$$
b a^{n} b^{-1} \neq a_{i}^{m}
$$

Then there is a homogeneous quasimorphism $h$ on $G$ such that

(1) $h(a)=1$, and $h\left(a_{i}\right)=0$ for all $i$,

(2) the defect of $h$ is $\leq C^{\prime}(\delta,|S|, T)$.

The weak version of the Gap Theorem follows from the strong version. Moreover, the first part of the strong version follows from the weak version together with an application of Bavard's theorem, but the implication in this direction uses the Axiom of Choice. Note that the constants $C$ appearing in the two versions are different in either case but related.

The second part of this strong version of the Gap Theorem can be thought of as a kind of separation theorem, which can be summarized in words as follows: given an element $a$ and a finite set of elements $a_{i} \in G$ whose nontrivial powers are never equal 
to a conjugate of a nontrivial power of $a$, one can (explicitly) construct a homogeneous quasimorphism that vanishes on all the $a_{i}$ and is positive on $a$; moreover, the defect of this quasimorphism can be uniformly controlled just in terms of the translation lengths of the $a_{i}$, and in terms of $\delta$ and $|S|$.

Such separation theorems have been pursued by several authors. For example, Polterovich and Rudnick [34] proved such a separation theorem for the group $\operatorname{SL}(2, \mathbb{Z})$, and asked if one can generalize it to hyperbolic groups; our work gives a quantitative positive answer to their question.

In $\S 6$ we state and prove the Accumulation Theorem:

Theorem B (Accumulation Theorem). Let $G$ be a torsion free non-elementary wordhyperbolic group. Then the first accumulation point $\delta_{\infty}$ for stable commutator length satisfies

$$
\frac{1}{12} \leq \delta_{\infty} \leq \frac{1}{2}
$$

Here $\delta_{\infty}$ is the first accumulation point for the values (with multiplicity) of the function scl on the set of conjugacy classes in $G$, thought of as an ordered subset of $\mathbb{R}$. As mentioned above, we think of this theorem as a kind of "homological Margulis Lemma". One can obtain similar theorems for groups with torsion, but the statement is not so clean or universal.

In $\S 7$ and $\S 8$ we discuss two important examples of groups acting on $\delta$-hyperbolic spaces: the action of the mapping class group on the complex of curves, and the action of an amalgamated free product on its associated tree.

Our main theorem about the mapping class group is:

Theorem C (Mapping Class Theorem). Let $S$ be a compact orientable surface of hyperbolic type and $\mathrm{MCG}(S)$ its mapping class group. Then there is a positive integer $P$ depending on $S$ such that for any pseudo-Anosov element a, either there is an $0<n \leq P$ and an element $b \in \operatorname{MCG}(S)$ with $b a^{-n} b^{-1}=a^{n}$, or else there exists a homogeneous quasimorphism $h$ on $\operatorname{MCG}(S)$ such that $h(a)=1$ and the defect of $h$ is $\leq C(S)$, where $C(S)$ depends only on $S$.

Moreover, let $a_{i} \in \operatorname{MCG}(S)$ be a collection of elements for which $T=\sup _{i} \tau\left(a_{i}\right)$ is finite. Suppose that for all integers $n, m \neq 0$ and all elements $b \in \operatorname{MCG}(S)$ and indices $i$ there is an inequality

$$
b a^{n} b^{-1} \neq a_{i}^{m}
$$

Then there is a homogeneous quasimorphism $h$ on $\operatorname{MCG}(S)$ such that

(1) $h(a)=1$, and $h\left(a_{i}\right)=0$ for all $i$,

(2) the defect of $h$ is $\leq C^{\prime}(S, T)$.

Note that if $b$ is not pseudo-Anosov, then $\tau(b)=0$. Moreover, for any surface $S$, there are infinitely many conjugacy classes of elements $b$ with $\tau(b) \leq O(1 / g \log (g))$ where $g=\operatorname{genus}(S)$ (see [15], especially Theorem 1.5). 
In the case of the mapping class group, one knows that not all the quasimorphisms are of the kind promised by this theorem. Along completely different lines, Endo and Kotschick [12] managed to obtain lower bounds on stable commutator length for elements $a \in S$ which are products of positive Dehn twists in disjoint curves. Such elements are reducible, and therefore fix a simplex in the complex of curves $\mathcal{C}(S)$; by contrast, our methods do not give any information about elements with fixed points.

Note that our arguments make use of a key acylindricity property proved by Bowditch, which is something like a bicombing in the context of $\mathcal{C}(S)$. Our lower bounds $d o$ depend on the surface $S$. There is some evidence that this dependence is necessary: a forthcoming paper by Kotschick [27] shows that stable commutator length goes to zero under stabilizing genus by adding handles.

Note that as in Theorem $\mathrm{A}^{\prime}$, Theorem $\mathrm{C}$ includes a separation theorem. Endo and Kotschick gave examples that set some limits on how far such a theorem might be generalized. Firstly, they show that there are elements $a \in \operatorname{MCG}(S)$ which are not pseudo-Anosov, but nevertheless for which there are homogeneous quasimorphisms which do not vanish on $a$. Secondly and conversely, they show that there are elements $b \in \operatorname{MCG}(S)$ of infinite order, for which no power of $b$ is conjugate to its inverse, but for which $h(b)=0$ for any homogeneous quasimorphism $h$ on $\operatorname{MCG}(S)$. These examples indicate the subtlety of the function $\operatorname{scl}$ on $\operatorname{MCG}(S)$, and suggest that a multiplicity of approaches are necessary to appreciate its full richness.

See [12] and [13] respectively for details.

Our main theorem about amalgamated free products is:

Theorem D (Amalgamation Theorem). Let $G=A *_{C} B$. Let $w$ be a word on $A, B$ which is reduced and cyclically reduced such that $|w|>1$, and let $\bar{w}$ denote the corresponding element in $G$. Suppose that the double coset $C \bar{w} C$ does not contain the element corresponding to any cyclic conjugate of $w^{-1}$. Then there exists a homogeneous quasimorphism $h$ on $G$ such that $h(\bar{w})=1$, and the defect of $h$ is $\leq 312$.

One can make a similar statement about HNN extensions.

Here a word $w$ is said to be cyclically reduced if $w$ is not equal to $v_{1} v_{2} v_{1}^{-1}\left(v_{1} \neq \emptyset\right)$ as a word. The double coset condition is at first glance somewhat odd. We examine a particular example (of essential slopes in knot complements in 3-manifolds) in some detail and show why it is natural and unavoidable.

1.2. Acknowledgments. We would like to thank Nathan Dunfield, Benson Farb, Étienne Ghys, Daniel Groves, Ursula Hamenstädt, Dieter Kotschick, Jason Manning, Shigenori Matsumoto, Igor Mineyev, Shigeyuki Morita, Leonid Polterovich and Bill Thurston. The second author appreciates the hospitality of the department of Mathematics at Caltech, where he visited the first author, and MPI in Bonn.

While writing this paper, the first author was partially supported by a Sloan Research Fellowship and NSF grant DMS-0405491. 


\section{Background Material}

2.1. $\delta$-hyperbolic groups and spaces. We assume that the reader is familiar with basic elements of the theory of $\delta$-hyperbolic groups and spaces: quasi-isometries, quasigeodesics, word-hyperbolicity, $\delta$-thin triangles, translation length, etc. For a reference, see [21].

Let $G$ be a $\delta$-hyperbolic group with a fixed generating set $S$. We denote the translation length of an element $g \in G$ on the Cayley graph $\Gamma_{S}(G)$ by $\tau(g)$. This length is defined by

$$
\tau(g)=\lim _{n \rightarrow \infty} \frac{\operatorname{dist}\left(\mathrm{id}, g^{n}\right)}{n},
$$

where distance is measured in the usual way in $\Gamma_{S}(G)$. Observe that $\tau$ is a class function.

If $g$ is torsion, $\tau(g)=0$. Otherwise, recall that there is a constant $N(|S|, \delta)$ and an integer $n<N$ such that a power $g^{n}$ of $g$ leaves invariant a bi-infinite geodesic axis and acts on this axis as translation through a distance $n \tau(g)$. Since this distance is necessarily a positive integer, this implies that there is a positive constant $B(|S|, \delta)$ such that $\tau(g) \geq B$ for every non-torsion element $g$. See e.g. Theorem 5.1 of [14].

\subsection{Stable commutator length}

Definition 2.1. Let $G$ be a group, and $a \in G$. The commutator length of $a$, denoted $\operatorname{cl}(a)$, is the minimum number of commutators whose product is equal to $a$. If $a$ is not in $[G, G]$, we $\operatorname{set} \operatorname{cl}(a)=\infty$. The stable commutator length, denoted $\operatorname{scl}(a)$, is the lim inf

$$
\operatorname{scl}(a)=\liminf _{n \rightarrow \infty} \frac{\operatorname{cl}\left(a^{n}\right)}{n} .
$$

Notice that $\operatorname{scl}(a)=\infty$ if and only if the order of $[a]$ in $H_{1}(G ; \mathbb{Z})$ is infinite.

The functions $\mathrm{cl}$ and $\mathrm{scl}$, like $\tau$, are class functions. Observe that $\mathrm{cl}$ and $\mathrm{scl}$ are monotone under homomorphisms. That is, if $\phi: G \rightarrow H$ is a homomorphism, then

$$
\operatorname{scl}(\phi(a)) \leq \operatorname{scl}(a)
$$

for all $a \in G$, and similarly for cl.

Example 2.2 (Mirror Condition). Let $G$ be a group and suppose that there are elements $a, b \in G$ and integers $n \neq m$ such that $b a^{n} b^{-1}=a^{m}$. Then

$$
n \cdot \operatorname{scl}(a)=\operatorname{scl}\left(a^{n}\right)=\operatorname{scl}\left(b a^{n} b^{-1}\right)=\operatorname{scl}\left(a^{m}\right)=m \cdot \operatorname{scl}(a)
$$

and therefore $\operatorname{scl}(a)=0$. (Note that the defining property of $a$ means that $a^{n-m} \in$ $[G, G], \operatorname{so} \operatorname{scl}(a) \neq \infty$.) 
Such elements can be found in Baumslag-Solitar groups. We compute

$$
|n| \cdot \tau(a)=\tau\left(a^{n}\right)=\tau\left(b a^{n} b^{-1}\right)=\tau\left(a^{m}\right)=|m| \cdot \tau(a),
$$

so if $\tau(a)$ is not zero (which can only happen for a torsion element when $G$ is hyperbolic), this is only possible if $n= \pm m$. The group

$$
\left\langle a, b \mid b a^{-1} b^{-1}=a, b^{2}=\mathrm{id}\right\rangle
$$

may be thought of as the (orbifold) fundamental group of the interval with mirrored endpoints, which is sometimes just called a mirror interval. In a hyperbolic manifold, an element of $\pi_{1}$ conjugate to its inverse is represented by a geodesic which "double covers" a geodesic segment with both endpoints on an orbifold stratum of order 2 (i.e., it double covers a mirror interval). Thus we will sometimes say that an element $a \in G$ for which there is no $b \in G$ and no integer $n \neq 0$ for which $b a^{n} b^{-1}=a^{-n}$ satisfies the no mirror condition.

Analogues of this condition will occur in the hypotheses of all our main theorems throughout this paper.

Example 2.3. Let $K$ be a knot complement in $S^{3}$ with genus $g$, and let $M_{p, q}$ be the result of $(p, q)$ Dehn filling on $K$. If $a \in \pi_{1}\left(M_{p, q}\right)$ represents the core of the added solid torus, then $\operatorname{scl}(a) \leq g / p$ (in fact, by taking covers and tubing boundary components together, one can actually obtain $\operatorname{scl}(a) \leq(g-1 / 2) / p$; see [5]). If $K$ is not a satellite knot or a torus knot, for all but finitely many pairs $(p, q)$ the resulting manifold $M_{p, q}$ is hyperbolic, and its fundamental group is word-hyperbolic. (See e.g. [35]).

\subsection{Quasimorphisms}

Definition 2.4. Let $G$ be a group. A quasimorphism is a function

$$
\phi: G \rightarrow \mathbb{R}
$$

for which there is a least constant $D(\phi) \geq 0$, called the defect, such that

$$
|\phi(a)+\phi(b)-\phi(a b)| \leq D(\phi)
$$

for all $a, b \in G$. In words, a quasimorphism fails to be linear by a bounded amount.

A quasimorphism is homogeneous if $\phi\left(a^{n}\right)=n \phi(a)$ for all integers $n$ and all $a \in G$.

If $\phi$ is a quasimorphism on $G$, then one can obtain a homogeneous quasimorphism $\bar{\phi}$ by the formula

$$
\bar{\phi}(a)=\lim _{n \rightarrow \infty} \frac{\phi\left(a^{n}\right)}{n} .
$$


Note that the defining property of a quasimorphism (that it is "almost linear") implies that the limit exists. A homogeneous quasimorphism is a class function.

The defect $D(\bar{\phi})$ is related to $D(\phi)$ by

$$
D(\bar{\phi}) \leq 2 \cdot D(\phi)
$$

see e.g. [6], Corollary 2.59. Homogeneous quasimorphisms are often easier to work with than ordinary quasimorphisms, but ordinary quasimorphisms are easier to construct. We use this averaging procedure to move back and forth between the two concepts.

We denote the vector space of all homogeneous quasimorphisms on $G$ by $Q(G)$. Observe that for any commutator $[a, b] \in G$ and any $\phi \in Q(G)$ we have

$$
|\phi([a, b])| \leq D(\phi) .
$$

It turns out that there is an equality

$$
\sup _{a, b}|\phi([a, b])|=D(\phi)
$$

see [1], Lemma 3.6.

Quasimorphisms and stable commutator length are related by Bavard's Duality Theorem (c.f. [1]):

Theorem 2.5 (Bavard's Duality Theorem). Let $G$ be a group. Then for any $a \in[G, G]$ we have an equality

$$
\operatorname{scl}(a)=\frac{1}{2} \sup _{\phi \in Q(G)} \frac{|\phi(a)|}{D(\phi)} .
$$

Note that one must take the supremum over $\phi \in Q(G)$ with $\phi(a) \neq 0$ (and therefore $D(\phi)>0$ ) for this to make sense; also, if $Q(G)=H^{1}(G)$ then $\operatorname{scl}(a)=0$ for $a \in[G, G]$. Note further that the theorem makes sense and is true for $a$ satisfying $a^{n} \in[G, G]$ for some positive $n$.

Bavard's theorem depends on the Hahn-Banach theorem and $L^{1}-L^{\infty}$ duality. Note that the Hahn-Banach theorem is equivalent to the Axiom of Choice. In particular, the quasimorphisms promised by Bavard's theorem are typically not constructible. Therefore we take explicit note in the sequel of when our arguments make use of Bavard's theorem and when they do not.

Example 2.6. For non-elementary hyperbolic groups $G$, the space $Q(G)$ has an uncountable dimension ([14]). But for certain groups, one can completely understand $Q(G)$. For example, let $G$ denote the universal central extension of the group of all orientation-preserving homeomorphisms of $S^{1}$. Then $Q(G)=\mathbb{R}$, generated by Poincaré's rotation number. In particular, every non-negative real number is equal to scl of some conjugacy class in this group. Similarly, let $\widehat{T}$ denote the universal central 
extension of Thompson's group $T$ of dyadic piecewise linear homeomorphisms of $S^{1}$. Then $\widehat{T}$ is a finitely presented group which realizes every non-negative rational number as scl. See [19] for more about the group $T$ and its bounded cohomology.

2.4. Mineyev's geodesic flow space. To understand stable commutator length in word-hyperbolic groups, one needs to control the geometry of maps of surfaces into $\delta$-hyperbolic spaces. Naively, following the usual practice in hyperbolic manifolds, one triangulates a surface and "straightens" the simplices, and then appeals to the Gauss-Bonnet theorem to control area and therefore diameter in terms of injectivity radius. In a $\delta$-hyperbolic space, the straightening must be done in a careful way. There are at least three technical approaches to this straightening:

(1) Gromov's geodesic flow space ([21], chapter 8),

(2) Mineyev-Monod-Shalom's homological ideal bicombing ([30]),

(3) Mineyev's geodesic flow space ([29]).

The approach in [21] is not entirely fleshed out, and the "geodesic flow" is really a quasigeodesic flow; therefore for our applications, Mineyev's flow space is best suited.

Mineyev constructs from a hyperbolic complex $X$ (for instance, a Cayley graph for a word-hyperbolic group) a geodesic flow space $\mathcal{F}(X)$, which consists of a union of parameterized lines joining ordered pairs of distinct points in the ideal boundary $\partial X$. The space $\mathcal{F}(X)$ admits a number of metrics and pseudo-metrics, of which the pseudo-metric $d^{\times}$is most important to us.

The following summarizes some of the main properties of $\mathcal{F}(X)$ which we use.

Theorem 2.7 (Mineyev). Let $X, d_{X}$ be a $\delta$-hyperbolic complex with valence $\leq n$. Then there exists a pseudo-metric space $\mathcal{F}(X), d^{\times}$called the flow space of $X$ with the following properties:

(1) $\mathcal{F}(X)$ is homeomorphic to $(\partial X \times \partial X-\Delta) \times \mathbb{R}$. The factors $(p, q, \cdot)$ under this homeomorphism are called the flowlines.

(2) There is an $\mathbb{R}$-action on $\mathcal{F}(X)$ (the geodesic flow) which acts as an isometric translation on each flowline $(p, q, \cdot)$.

(3) There is a $\mathbb{Z} / 2 \mathbb{Z}$ action $x \rightarrow x^{*}$ which anti-commutes with the $\mathbb{R}$ action, which satisfies $d^{\times}\left(x, x^{*}\right)=0$, and which interchanges the flowlines $(p, q, \cdot)$ and $(q, p, \cdot)$.

(4) There is a natural action of $\operatorname{Isom}(X)$ on $\mathcal{F}(X)$ by isometries. If $g \in \operatorname{Isom}(X)$ is hyperbolic with fixed points $p^{ \pm}$in $\partial X$ then $g$ fixes the flowline $\left(p^{-}, p^{+}, \cdot\right)$ of $\mathcal{F}(X)$ and acts on it as a translation by a distance which we denote $\tau(g)$. This action of $\operatorname{Isom}(X)$ commutes with the $\mathbb{R}$ and $\mathbb{Z} / 2 \mathbb{Z}$ actions.

(5) There are constants $M \geq 0$ and $0 \leq \lambda<1$ such that for all triples $a, b, c \in \partial X$, there is a natural isometric parameterization of the flowlines $(a, c, \cdot),(b, c, \cdot)$ 
for which there is exponential convergence

$$
d^{\times}((a, c, t),(b, c, t)) \leq M \lambda^{t} .
$$

(6) If $X$ admits a cocompact isometric action, and $G \subset \operatorname{Isom}(X)$ is torsion free, then there is a $G$-equivariant $(K, \epsilon)$ quasi-isometry between $\mathcal{F}(X), d^{\times}$and $X, d_{X}$.

Moreover, all constants as above depend only on $\delta$ and $n$.

Note that although $\mathcal{F}(X)$ is homeomorphic to $(\partial X \times \partial X-\Delta) \times \mathbb{R}$, this topology is not induced by the pseudo-metric $d^{\times}$, since this pseudo-metric fails to separate pairs of points interchanged by the $\mathbb{Z} / 2 \mathbb{Z}$ action.

This is a conflation of several results in [29]. The pseudo-metric $d^{\times}$is defined in Section 3.2 and Section 8.6 on a slightly larger space which Mineyev calls the symmetric join. The flow space, defined in Section 13, is a natural subset of this. The basic properties of the $\mathbb{R}, \mathbb{Z} / 2 \mathbb{Z}$ and $\operatorname{Isom}(X)$ action are proved in Section 2. The remaining properties are subsets of Theorem 44 (p. 459) and Theorem 57 (p. 468). There is another natural metric $d_{*}$ on $\mathcal{F}(X)$ which induces the topology on $\mathscr{F}(X)$, and for which the $\mathbb{R}$ action is by bi-Lipschitz homeomorphisms.

For our applications, the key points are that the action of hyperbolic isometries on the flowlines is by translations, and the exponential convergence of flowlines with common endpoints at infinity.

Remark 2.8. Since the flowlines $(p, q, \cdot)$ and $(q, p, \cdot)$ are distance 0 apart in $d^{\times}$, we may think of them as different parameterizations of the same lines in a suitable quotient on which $d^{\times}$is a metric (and not just a pseudo-metric).

Remark 2.9. In bullet (5), the synchronous parameterizations of ( $a, c, t)$ and $(b, c, t)$ for which exponential convergence holds are precisely those for which $(a, c, 0)$ is the point on the flowline $(a, c, \cdot)$ closest to $b$, and $(b, c, 0)$ is the point on the flowline $(b, c, \cdot)$ closest to $a$ (interpreted in terms of suitable horofunctions). In particular, for every triple $a, b, c$ of distinct points in $\mathscr{F}(X)$, if $\Delta$ is a triangle obtained from the union of three flowlines joining these points in pairs, the edges of $\Delta$ are exponentially close to each other away from a compact subset of $\Delta$ of uniformly bounded diameter (i.e., the diameter of the "thick part" of $\Delta$ is bounded independently of the choice of $a, b, c)$.

\section{The Gap Theorem, first version}

The Gap Theorem, to be proved below in its first version, says that in a wordhyperbolic group $G$, if $a$ is an element which satisfies the no mirror condition (from 
Example 2.2) and has a sufficiently long translation length, then the stable commutator length of $a$ can be uniformly bounded from below. Example 2.2 and Example 2.3 together point to the necessity of both hypotheses.

Theorem A (Gap Theorem, weak version). Let $G$ be a word-hyperbolic group that is $\delta$-hyperbolic with respect to a symmetric generating set $S$ with $|S|$ generators. Then there is a constant $C(\delta,|S|)>0$ such that for every $a \in G$, either $\operatorname{scl}(a) \geq C$ or else there is some positive integer $n$ and some $b \in G$ such that $b a^{-n} b^{-1}=a^{n}$.

Remark 3.1. By Bavard's theorem, the uniform lower bound on $\operatorname{scl}(a)$ is equivalent to the existence of a homogeneous quasimorphism $\phi$ with $\phi(a)=1$ for which there is a uniform upper bound on the defect $D(\phi)$ depending only on $\delta$ and $|S|$.

Remark 3.2. If $b a^{-n} b^{-1}=a^{n}$ for some $b$ and positive integer $n$, then $b^{2}$ and $a^{n}$ commute, and since $G$ is hyperbolic, suitable powers of $a$ and $b$ generate an infinite dihedral group.

It follows that if $G$ is torsion free, no such element $b$ can exist. Conversely, note that every torsion element $a$ satisfies $a^{n}=a^{-n}=$ id for some positive $n$. It follows that the no mirrors condition is vacuously satisfied in a torsion-free hyperbolic group.

The following proof uses properties of Mineyev's geodesic flow space. We prove a stronger theorem in $\S 5$, by directly constructing sufficiently many quasimorphisms. The second proof is logically superior to the first, since the construction is direct, and moreover the existence of these quasimorphisms does not depend on Bavard's Theorem and the Axiom of Choice. However, the first proof is more "geometric".

Proof. Let $\Gamma_{S}(G)$ denote the Cayley graph of $G$ with respect to the generating set $S$. For any element $a \in G$ recall that $\tau(a)$ denotes the translation length of $a$. As observed in $\S 2.1$, every element $a \in G$ is either torsion, or has a power $a^{n}$ where $n \leq C_{2}(|S|, \delta)$ that fixes an axis $l_{a}$, and therefore has $\tau(a) \geq C_{1}(|S|, \delta)$. For the remainder of the proof we replace $a$ by a suitable power $a^{n}$, and assume that $a$ fixes an axis $l_{a}$.

For convenience, we let $K$ be a 2-complex coming from a finite presentation for $G$ with generating set $S$. Observe that $\Gamma_{S}(G)$ is the 1-skeleton of the universal cover $\tilde{K}$. Suppose that $\operatorname{scl}(a)$ is very small so that there is an expression

$$
a^{n}=\prod_{i=1}^{m}\left[b_{i}, c_{i}\right]
$$

for some $b_{i}, c_{i} \in G$ such that $m / n$ is small. Let $\Sigma$ be a surface of genus $m$, and $\sigma: \Sigma \rightarrow K$ a simplicial map which takes the boundary to the immersed circle $\gamma=$ $l_{a} / a^{n}$. We would like to choose a representative surface whose area and geometry can be controlled by $\chi(\Sigma)$ and length $(\gamma)$. Since we need to control constants, this must be done carefully. 
We appeal to Mineyev's Theorem 2.7. Using this theorem, one proceeds as follows. One picks a 1-vertex triangulation of $\Sigma$, and spins the vertex along $\partial \Sigma$ thereby producing an ideal triangulation whose edges can be realized as a subset in the quotient space $\mathcal{F}\left(\Gamma_{S}(G)\right) / G$. This is covered by a $\pi_{1}(\Sigma)$ equivariant map from the 1-skeleton of $\widetilde{\Sigma}$ to $\mathcal{F}\left(\Gamma_{S}(G)\right)$, where $\pi_{1}(\Sigma)$ acts on $\mathcal{F}\left(\Gamma_{S}(G)\right)$ by its image in $G$. Each ideal triangle in $\widetilde{\Sigma}$ corresponds to a union of three flowlines in $\mathcal{F}\left(\Gamma_{S}(G)\right)$.

In $\mathcal{F}$, as pointed out in Remark 2.9, each triangle consists of a thick core of diameter at most $C_{3}$ (depending only on $\delta$ and $|S|$ ) together with thin regions consisting of pairs of geodesic rays whose distance converges exponentially fast to zero. The image of $\Sigma$ consists of $2|\chi(\Sigma)|=4 m-2$ triangles. Because of the exponential convergence of geodesics in $\mathcal{F}$, we can give $\Sigma$ a hyperbolic metric of constant curvature $-\kappa$ in such a way that the triangles are totally geodesic, and the map to $\mathcal{F}\left(\Gamma_{S}(G)\right)$ is distance decreasing on the 1-skeleton, where $\kappa>0$ depends only on $\delta$ and $|S|$. It follows from the Gauss-Bonnet theorem that away from a thick part consisting of at most $(4 m-2)$ regions whose diameters sum to at most $(4 m-2) C_{3}$ for some constant $C_{3}$, the thickness of $\Sigma$ is bounded by a constant $C_{4}$, where $C_{3}$ and $C_{4}$ depend only on $|S|$ and $\delta$. In fact, by choosing $C_{3}$ sufficiently large, we may assume that $C_{4}$ is as small as we like, a fact which we will not use.

After composing with a quasi-isometry

$$
\phi: \mathcal{F}(X) \rightarrow \widetilde{K}
$$

and filling in the map on triangles, we get an induced map

$$
\tilde{\sigma}_{1}: \tilde{\Sigma} \rightarrow \tilde{K}
$$

It does not really matter how the map is filled in on triangles, since we are ultimately only interested in the distances between points contained in the image of $\partial \widetilde{\Sigma}$. Again, by Theorem 2.7, we can assume that the quasi-isometry constants of $\phi$ depend only on $\delta$ and $|S|$.

Under this quasi-isometry, the constants $C_{3}$ and $C_{4}$ for the diameter of the core and the thickness of the complementary region must be replaced by analogous constants $C_{3}^{\prime}$ and $C_{4}^{\prime}$ which still depend only on $\delta$ and $|S|$. The image of every boundary component is a quasigeodesic which is within a bounded distance of some translate of the axis $l_{a}$; it follows that for a suitable choice of $\phi$, without changing the constants involved, we can assume that $\widetilde{\Sigma}_{1}$ takes $\partial \widetilde{\Sigma}$ in a $\pi_{1}(\Sigma)$-equivariant way to a union of translates of $l_{a}$.

By abuse of notation, we denote the image of this map as $\widetilde{\Sigma}_{1}$. Let $\alpha$ be a fundamental domain for the action of $a^{n}$ on $l_{a}$. By our estimates, away from a subset of $\alpha$ of length at most $(4 m-2) C_{3}^{\prime}$, every point $p \in \alpha$ can be joined by an arc $\tilde{\beta}_{p}$ in the image of $\widetilde{\Sigma}_{1}$, such that the endpoints of $\tilde{\beta}_{p}$ are a distance at most $C_{4}^{\prime}$ apart in $\tilde{K}$, and lie on distinct components $l_{a}$ and $l_{a}^{i}$ of $\widetilde{\Sigma}_{1}\left(\partial \widetilde{\Sigma}_{1}\right)$. Pulling back by $\widetilde{\Sigma}_{1}$ and projecting to $\Sigma_{1}$, we obtain a homotopically essential proper arc $\beta_{p} \in \Sigma_{1}$. If $p_{1}, p_{2}$ are at least distance $2 C_{4}^{\prime}$ apart in $\alpha$, we see that the pulled-back arcs $\beta_{p_{1}}, \beta_{p_{2}}$ can be isotoped to 
be disjoint in $\Sigma_{1}$. A surface of genus $m$ with one boundary component contains at most $6 m-3$ disjoint nonparallel proper essential arcs, and each arc has 2 endpoints. It follows that there are at most $12 m-6$ components $l_{a}^{i}$ which are joined by $\operatorname{arcs} \tilde{\beta}_{p}$ with nearby endpoints to points in $\alpha$.

For a fixed component $l_{a}^{i}$, the set of points where $l_{a}^{i}$ is close to $l_{a}$ is (coarsely) connected, by convexity of quasigeodesics in $\delta$-hyperbolic spaces. Consequently there is a connected arc $\sigma^{\prime} \subset \alpha$ satisfying

$$
\text { length }\left(\sigma^{\prime}\right) \geq \frac{\text { length }(\alpha)-(4 m-2)\left(C_{3}^{\prime}+C_{4}^{\prime}\right)}{12 m-6},
$$

which cobounds a strip $R^{\prime}$ of $\widetilde{\Sigma}_{1}$ of width $\leq C_{4}^{\prime}$ with some fixed $l_{a}^{i}$.

By convexity of quasigeodesics, there is a connected subinterval $\sigma \subset \sigma^{\prime}$ satisfying

$$
\text { length }(\sigma) \geq \operatorname{length}\left(\sigma^{\prime}\right)-2 C_{4}^{\prime}
$$

such that $\sigma$ cobounds a strip $R$ of $\widetilde{\Sigma}_{1}$ of width $C_{5}$ depending only on $\delta$ with some fixed $l_{a}^{i}$. The strip $R$ is much longer than it is wide, and it makes sense to say that a choice of orientations on the $l_{a}$ and $l_{a}^{i}$ agree or disagree (see Figure 1):
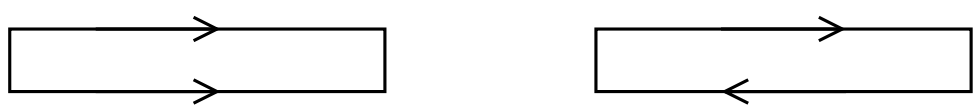

Figure 1. The orientations on $l_{a}$ and $l_{a}^{i}$ might agree or disagree along $R$.

Now, since $\Sigma_{1}$ is oriented, the induced orientations on $l_{a}$ and $l_{a}^{i}$ disagree. Therefore $l_{a}^{i}$ is a nontrivial translate of the axis $l_{a}$. That is, there is some $c$ with $l_{a}^{i}=c\left(l_{a}\right)$. Then $e=c a^{-1} c^{-1}$ stabilizes $l_{a}^{i}$, and moves points in (roughly) the same direction as $a$.

Let $p$ be the midpoint of $\sigma$. Suppose that length $(\gamma)=\tau(a)$ is big compared to the constant $C_{5}$ (e.g., length $(\gamma) \geq 2 C_{5}$ will do). We consider the translates $e^{-w} a^{w}(p)$ for

$$
|w| \leq \frac{\operatorname{length}(\sigma)}{3 \cdot \operatorname{length}(\gamma)} \leq \frac{n}{36 m}+1
$$

where the last inequality follows from our assumptions about length $(\gamma)$.

Then by our estimate on the width of the strip $R$, we have

$$
d\left(p, e^{-w} a^{w}(p)\right) \leq 4 C_{5} .
$$

There are at most $|S|^{4 C_{5}}$ elements in the group which move the point $p$ a distance $\leq 4 C_{5}$, and therefore if

$$
\frac{n}{36 m}+1>|S|^{4 C_{5}}
$$

then by the pigeonhole principle there are $w_{1} \neq w_{2}$ such that

$$
e^{-w_{1}} a^{w_{1}}=e^{-w_{2}} a^{w_{2}}
$$


and therefore

$$
c a^{w_{1}-w_{2}} c^{-1}=e^{w_{2}-w_{1}}=a^{w_{2}-w_{1}} .
$$

In words: if $\operatorname{scl}(a)$ is too small, a nontrivial power of $a$ is conjugate to its inverse.

It follows contrapositively that when $\tau(a) \geq 2 C_{5}$ and no power of $a$ is conjugate to its inverse, we have $\operatorname{scl}(a) \geq \frac{1}{36}|S|^{-4 C_{5}}$. If $a$ is not torsion, $\tau(a) \geq C_{1}$. So for any $a$ with no power conjugate to its inverse, we have an estimate

$$
\operatorname{scl}\left(a^{2 C_{5} / C_{1}}\right) \geq \frac{1}{36}|S|^{-4 C_{5}} .
$$

Therefore the constant

$$
C(\delta,|S|)=\frac{1}{36}|S|^{-4 C_{5}} \frac{C_{1}}{2 C_{5}}
$$

satisfies the claim of the theorem.

Remark 3.3. It follows from the proof that if $a$ does not satisfy the no mirror condition, so that there is a positive $n$ with $a^{n}=c a^{-n} c^{-1}$ for some $c$, then $n$ may be chosen to be less than some constant depending only on $\delta$ and $|S|$.

It is interesting to ask the following:

Question 3.4. Let $G$ be a group, and let $H$ be the set of elements in $G$ which satisfy the no mirror condition, i.e., for which $a^{n} \neq b a^{-n} b^{-1}$ whenever $b \in G$ and $n \neq 0$. Under what circumstances is $\inf _{a \in H} \operatorname{scl}(a)$ positive?

We say that a group $G$ has the stable commutator gap property if it has the property described in Question 3.4. Theorem A shows that hyperbolic groups have the stable commutator gap property.

Example 3.5. In [7], Comerford and Edmunds show that in a free group $F$, every nonzero element $a$ satisfies $\operatorname{scl}(a) \geq 1 / 2$ (this lower bound essentially follows from an earlier result of Duncan and Howie [10] and improves an earlier estimate $\operatorname{scl}(a) \geq 1 / 6$ obtained by Culler in [8]). Let $G$ be residually free; i.e., suppose that for all nonzero $a \in G$ there is a homomorphism $\phi_{a}: G \rightarrow F$ to a free group, for which $\phi_{a}(a)$ is nonzero. Since scl is nonincreasing under homomorphisms, $G$ satisfies the stable commutator gap property.

\section{Counting functions}

Here we review so-called counting functions, which generalize a construction introduced by Brooks [4] to construct quasimorphisms on free groups. See [14], [16] or [2] for more details. 
Suppose that $G$ is a group with a fixed symmetric generating set $S$, and $\Gamma:=$ $\Gamma_{S}(G)$ is its Cayley graph. Let $w$ be a word in the generating set. Let $\alpha$ be a (directed) path in $\Gamma$, and $|\alpha|$ its length. Define $|\alpha|_{w}$ to be the maximal number of times that $w$ can be seen as an (oriented) subword of $\alpha$ without overlapping.

Example 4.1. $|x y x y x|_{x y}=2$. $|x y x y x|_{x y x}=1 .|x x y x y|_{y x}=1$.

Let $0<W<|w|$ be a constant. For $x, y \in \Gamma$, define

$$
c_{w, W}(x, y):=d(x, y)-\inf _{\alpha}\left(|\alpha|-W|\alpha|_{w}\right),
$$

where $\alpha$ ranges over all the paths from $x$ to $y$. If the infimum is attained by $\alpha$, we say that $\alpha$ is a realizing path for $c_{w, W}$ from $x$ to $y$. If $\gamma$ is a geodesic from $x$ to $y$, then define $c_{w, W}(\gamma)=c_{w, W}(x, y)$.

Fix a point $x \in \Gamma$. Define, for $a \in G$,

$$
c_{w, W}(a):=c_{w, W}(x, a x) .
$$

$c_{w, W}$ is called the counting function for the pair $(w, W)$. Let $w^{-1}$ denote the inverse word of $w$. We define

$$
h_{w, W}:=c_{w, W}-c_{w^{-1}, W}
$$

In [14], the normalization $W=1$ is used. This is an appropriate choice of constant when $w^{*}:=\ldots w w w w \ldots$ is a bi-infinite geodesic, which is the case in our applications throughout this paper.

More generally, when $G$ acts on a graph $\Gamma$ (not necessarily properly), we modify the definition of counting functions as follows. Let $w$ be a path in $\Gamma$ and call $a w$ for $a \in G$ a copy of $w$ or (interchangeably) a translate of $w$. For a path $\alpha$ in $\Gamma$, define $|\alpha|_{w}$ to be the maximal number of disjoint oriented copies of $w$ which can be obtained as subpaths of $\alpha$. All other definitions are as above. These modified counting functions will be important in the sequel when we discuss the action of the mapping class group $\operatorname{MCG}(S)$ on the complex of curves $\ell(S)$, for some surface $S$.

Proposition 4.2 (Lemma 3.3 [16]). If $\alpha$ is a realizing path for $c_{w, W}$, then it is a $(K, \epsilon)$-quasigeodesic, where

$$
K=\frac{|w|}{|w|-W}, \quad \epsilon=\frac{2 W|w|}{|w|-W} .
$$

It is known that in a $\delta$-hyperbolic space any two $(K, \epsilon)$-quasigeodesics which have same end points stay in the $L(K, \epsilon, \delta)$-neighborhood of each other for some universal constant $L$ (see [21]).

Let $L=L(|w| /(|w|-W), 2 W|w| /(|w|-W), \delta)$. Let $\alpha$ be a geodesic from $x$ to $y$. From Proposition 4.2 we deduce that a realizing path for $\alpha$ must be contained in the $L$-neighborhood of $\alpha$. Consequently, if the $L$-neighborhood of $\alpha$ does not contain a copy of $w$, then $c_{w, W}(\alpha)=0$. 
Remark 4.3. We will use this fact later in our argument to avoid "reverse counting". Roughly speaking, let $w$ be a word such that $w^{n}$ is a geodesic. Then, for $n>0$,

$$
c_{w, W}\left(w^{n}\right) \geq W n
$$

because $\left|w^{n}\right|_{w}=n$.

Suppose that the $L$-neighborhood of $w^{n}$ does not contain a copy of $w^{-1}$. Here we are thinking of the $L$-neighborhood of $w^{n}$, for large $n$, like a long narrow tube whose core has a definite orientation, agreeing with the orientation on $w$. By "a copy of $w^{-1}$ " here we mean a copy of $w$ whose orientation disagrees with that of the core of the tube (compare with Figure 1 and the accompanying discussion). Then it follows that $c_{w^{-1}, W}\left(w^{n}\right)=0$ because for a realizing path $\alpha$ for $c_{w^{-1}, W}$ at $w^{n}$ we must have $|\alpha|_{w^{-1}}=0$. We thus obtain for all $n>0$ an inequality $h_{w, W}\left(w^{n}\right) \geq n W$.

Let $D(h)$ be the defect of $h$. Then we have the following inequality:

Proposition 4.4 (Proposition 3.10 [16]).

$$
D\left(h_{w, W}\right) \leq 12 L+6 W+48 \delta \text {. }
$$

Remark 4.5. Note that the defect only depends on $|w|, W, \delta$. If we take $W=1$, then $L$ depends only on $\delta$ if $|w| \geq 2$. In particular, the upper bound in Proposition 4.4 depends only on $\delta$. We will take $W=1$ in all the applications in this paper.

\section{The Gap Theorem, improved version}

We are now in a position to state and prove the improved version of the Gap Theorem, using counting quasimorphisms. The following theorem improves Theorem A in at least two ways: it does not use Bavard's theorem or Mineyev's flow space, and the constants in questions can be effectively estimated.

Theorem A' (Gap Theorem, strong version). Let $G$ be a word-hyperbolic group that is $\delta$-hyperbolic with respect to a symmetric generating set $S$ with $|S|$ generators. Let $a \in G$ be a (non-torsion) element. Assume that there is no $n>0$ and no $b \in G$ with $b a^{-n} b^{-1}=a^{n}$. Then there is a homogeneous quasimorphism $h$ on $G$ such that

(1) $h(a)=1$,

(2) the defect of $h$ is $\leq C(\delta,|S|)$.

Moreover, let $a_{i} \in G$ be a collection of elements for which $T=\sup _{i} \tau\left(a_{i}\right)$ is finite. Suppose that for all integers $n, m \neq 0$ and all elements $b \in G$ and indices $i$ there is an inequality

$$
b a^{n} b^{-1} \neq a_{i}^{m}
$$

Then there is a homogeneous quasimorphism $h$ on $G$ such that 
(1) $h(a)=1$, and $h\left(a_{i}\right)=0$ for all $i$,

(2) the defect of $h$ is $\leq C^{\prime}(\delta,|S|, T)$.

Remark 5.1. Note that in the second part of the theorem, we must state a generalization of the no mirror condition, involving both $a$ and the elements $a_{i}$.

Remark 5.2. Hamenstädt ([24]) used dynamical methods to directly construct quasimorphisms on word-hyperbolic groups. Similar ideas were developed by Picaud ([32]) in the special case of surface groups. It is not clear to us how to use these methods to obtain uniform estimates on defects.

Proof. We follow [14], [16]. We assume in what follows that $a$ is not torsion. Let $N$ be a constant such that $a^{N}$ stabilizes a bi-infinite geodesic $l_{a}$. Note that one may find an $N$ whose size can be bounded in terms of $\delta$ and $|S|$. We also choose $N$ so that $N \tau(a) \gg L(\delta)$. Note that since $\tau(a)$ can be bounded from below by a positive constant depending only on $\delta$ and $|S|$, the constant $N$ still depends only on $\delta$ and $|S|$ (see the proof of Theorem A).

After replacing $a$ by a conjugate if necessary, we may assume that $1 \in l_{a}$. Set $b=a^{N} . l_{a}$ is an axis for $b$. We denote the subpath of $l_{a}$ from 1 to $b$ by $l_{a} \mid b$ and the subpath from 1 to $b^{-1}$ by $l_{a} \mid b^{-1}$. Note that we think of these as oriented segments.

Now let $c_{b}$ be the counting function on $G$ for the pair $\left(l_{a} \mid b, 1\right)$ (note that we are setting $W=1$ in the notation of $\S 4$ ). Then for any $n>0$, we obtain an estimate $c_{b}\left(b^{n}\right) \geq n$, since the subpath of $l_{a}$ from 1 to $b^{n}$ can be tiled by $n$ disjoint translates of $l_{a} \mid b$. At the cost of possibly replacing $N$ by $2 N$ if necessary, we may assume that $\left|l_{a}\right| b \mid \geq 2$, so that $L$ (as in $\S 4$ ) depends only on $\delta$.

Claim 1: There is a constant $C_{1}(\delta,|S|)$ such that if $N \geq C_{1}$ then there is no translate of $l_{a} \mid b^{-1}$ in the $L$-neighborhood of $l_{a}$ whose orientation agrees with that of $l_{a}$.

Note that $l_{a} \mid b^{-1}$ is a copy of $l_{a} \mid b$ with the opposite orientation, so we could just as well state Claim 1 as saying that there is no translate of $l_{a} \mid b$ in the $L$-neighborhood of $l_{a}$ whose orientation disagrees with that of $l_{a}$. We will prove the claim later; we call the conclusion of the claim "no reverse counting", and prove our theorem under this hypothesis.

Take $N$ to satisfy $N \geq C_{1}$ as well in the following. Note that $N$ can still be chosen satisfying these criteria with size bounded from above in terms of $\delta$ and $|S|$.

Let $c_{b^{-1}}$ be the counting function for the pair $\left(l_{a} \mid b^{-1}, 1\right)$. It follows from Claim 1 that

$$
c_{b^{-1}}\left(b^{n}\right)=0
$$

for all $n>0$. We define $h_{b}=c_{b}-c_{b^{-1}}$ and then obtain

$$
h_{b}\left(b^{n}\right) \geq n
$$

for any $n>0$. 
By Proposition 4.4 and Remark 4.5, there is a constant $K(\delta)$, which depends only on $\delta$ such that

$$
D\left(h_{b}\right) \leq K(\delta) .
$$

Since $b=a^{N}$, we get $h_{b}\left(a^{n}\right) \geq n / N$ for all $n>0$. By averaging, we may replace $h_{b}$ by a homogeneous quasimorphism $h^{\prime}=\bar{h}_{b}$ (see $\S 2.3$ ). Then $D\left(h^{\prime}\right) \leq 4 K(\delta)$ and $h^{\prime}(a) \geq 1 / N$. Define $h=m h^{\prime}$ for some constant $m$ for which $h(a)=1$. Then $h$ is a homogeneous quasimorphism, and satisfies $D(h) \leq 4 N K(\delta)$.

This proves the first part of the theorem, modulo Claim 1.

The second part follows by almost the same argument. First of all, we may assume that $a_{i}$ has infinite order (equivalently, $\tau\left(a_{i}\right)>0$ ) since every homogeneous quasimorphism will already vanish on torsion. Since we are looking for a homogeneous quasimorphism which vanishes on $a_{i}$, without loss of generality, we may replace each $a_{i}$ by a conjugate of a non-trivial power $(\leq N(\delta,|S|))$. Therefore, we may assume that $a_{i}$ has a geodesic axis $l_{a_{i}}$, with $1 \in l_{a_{i}}$.

Let $v$ be an oriented geodesic path in $\Gamma_{S}(G)$ from 1 to $a^{N}$, and let $v^{-1}$ be $v$ with the opposite orientation. In other words, $v=\left.l_{a}\right|_{a^{N}}$.

A relative version of Claim 1 proves the second part of the theorem. We give two different proofs, which give different constants. Recall our notation $b=a^{N}$.

Claim 2: Suppose that $N$ satisfies

$$
N \geq C_{1}|S|^{\tau(a)} \frac{\tau(a)}{\tau\left(a_{i}\right)+1}
$$

for suitable $C_{1}$ depending only on $\delta$ and $|S|$. Then there is no copy of $l_{a} \mid b$ or $l_{a} \mid b^{-1}$ in the $L$-neighborhood of $l_{a_{i}}$.

Claim 2': Suppose that $N$ satisfies

$$
N \geq C_{1}|S|^{T}
$$

for suitable $C_{1}$ depending only on $\delta$ and $|S|$. Then there is no copy of $l_{a} \mid b$ or $l_{a} \mid b^{-1}$ in the $L$-neighborhood of $l_{a_{i}}$.

It follows from Claim 2 or $2^{\prime}$ that $h$ as constructed above satisfies $h\left(a_{i}\right)=0$ for all $i$.

Proof of Claim 1. In fact, the proof of the claim follows by the same argument as the end of the proof of Theorem A. A copy of $l_{a} \mid b^{-1}$ in the $L$-neighborhood of $l_{a}$ is contained in an axis of some element $e$ which is conjugate to $a^{-1}$; i.e. $e=c a^{-1} c^{-1}$ for some $c$.

Let $v$ denote such a copy of $l_{a} \mid b^{-1}$, and let $v_{0}$ represent its initial point. The geodesic $l_{a}$ is invariant under $a^{N}$ but not necessarily under $a$ itself. Nevertheless, there is a constant $C_{2}$ depending only on $\delta$ such that $a^{i}\left(v_{0}\right)$ is within distance $C_{2}$ of $l_{a}$, for any integer $i$. Then for all $i<N$ the element $e^{-i} a^{i}$ satisfies

$$
d\left(v_{0}, e^{-i} a^{i}\left(v_{0}\right)\right) \leq 2 L+2 C_{2} .
$$


So if $N>|S|^{2 L+2 C_{2}}$ we must have $e^{-i_{1}} a^{i_{1}}=e^{-i_{2}} a^{i_{2}}$ for two distinct indices $i_{1}, i_{2}$, and therefore the $\left(i_{2}-i_{1}\right)$-th power of $a$ is conjugate by $c$ to its inverse. Set $C_{1}=|S|^{2 L+2 C_{2}}$.

Proof of Claim 2. We only sketch the proof of Claim 2 since the details are almost identical to those of the proof of Claim 1 . If the $L$-neighborhood of $l_{a_{i}}$ contains a copy of a arbitrarily long subpath of $l_{a}$, we can find a point $v_{0}$ for which

$$
d\left(v_{0}, e^{k_{j}} a_{i}^{j}\left(v_{0}\right)\right) \leq 2 L+2 C_{2}+\tau(a)
$$

for some conjugate $e$ of $a$ or $a^{-1}$, and for any $0 \leq j \leq J$ for an arbitrarily big (fixed) $J$. It follows that if $N>|S|^{2 L+2 C_{2}+\tau(a)}$ then some non-trivial power of $a_{i}$ is conjugate to some (possibly trivial) power of $a$, say $a^{k}$. Moreover, if

$$
N>|S|^{2 L+2 C_{2}+\tau(a)} \frac{\tau(a)}{\tau\left(a_{i}\right)+1}
$$

then $k \neq 0$, contrary to hypothesis.

Proof of Claim 2'. This argument interchanges the roles of $a$ and $a_{i}$ in the proof of Claim 2. Suppose that the $L$-neighborhood of $l_{a_{i}}$ contains a copy, $v$, of $l_{a} \mid b$ or $l_{a} \mid b^{-1}$. The segment $v$ is a part of an axis of $e=c a c^{-1}$ or $c a^{-1} c^{-1}$. Let $v_{0}$ be the starting point of $v$. Then for each $0 \leq j \leq N$, there exists $k_{j}$ such that

$$
d\left(v_{0}, a_{i}^{k_{j}} e^{j}\left(v_{0}\right)\right) \leq 2 L+2 C_{2}+\tau\left(a_{i}\right) \leq 2 L+2 C_{2}+T
$$

(we use the same constant $C_{2}$ for $a_{i}$ ). Therefore, if $N>|S|^{2 L+2 C_{2}+T}$, then some non-trivial power of $a$ is conjugate to some (possibly trivial) power of $a_{i}$, which is impossible.

This completes the proof of the theorem.

Remark 5.3. The second statement can be used to give a lower bound of the stable commutator length "relative to $\left\{a_{i}\right\}$ ". That is, if we can write $a$ as a product

$$
a=\left[b_{1}, c_{1}\right] \ldots\left[b_{n}, c_{n}\right] a_{i_{1}}^{m_{1}} \ldots a_{i_{k}}^{m_{k}}
$$

then we say the relative length of $a$ is $\leq n+k / 2$. The infimum of this number is the relative commutator length, and the liminf of the relative commutator length of $a^{n}$ divided by $n$ as $n \rightarrow \infty$ is the relative stable commutator length. Using our theorem, the relative commutator length has a lower bound of $1 / 2 D(h)$, where $h$ is a homogeneous quasimorphism obtained in the second part for $a$ and the $a_{i}$.

Remark 5.4. One may reinterpret the second part of Theorem $\mathrm{A}^{\prime}$ as follows. In any group $G$, let $B_{1}(G)$ denote the real vector space of group 1-boundaries (i.e., the group 
1-cycles that are boundaries of group 2-chains), and for any chain $\sum t_{i} g_{i} \in B_{1}(G)$ define scl by the formula

$$
\operatorname{scl}\left(\sum t_{i} g_{i}\right)=\sup _{\phi} \frac{\left|\sum t_{i} \phi\left(g_{i}\right)\right|}{2 D(\phi)}
$$

(compare with Theorem 2.5). This function is a pseudo-norm, and agrees with scl on ordinary elements (see [6], §2.6). It evidently vanishes on the subspace $H$ spanned by cycles of the form $g-h g h^{-1}$ and $g^{n}-n g$, and descends to a quotient pseudonorm on $B_{1}^{H}(G):=B_{1}(G) / H$. Then the second part of Theorem $\mathrm{A}^{\prime}$ implies that whenever $G$ is hyperbolic, scl is a genuine norm on $B_{1}^{H}(G)$.

We refer to the homogeneous quasimorphisms constructed in the proof of Theorem $\mathrm{A}^{\prime}$ as counting quasimorphisms, by contrast with the abstract quasimorphisms promised by Bavard's theorem.

It is interesting to speculate that one could use Theorem A or $\mathrm{A}^{\prime}$ as a starting point to invert the word-hyperbolic Dehn surgery theory developed by Groves and Manning and independently by Osin (cf. [23], [31]).

Corollary 5.5. Let $K$ be a knot in $S^{3}$ of genus $g$. Then for any $\delta>0$ and every integer $n>0$ there is a constant $C(\delta, n)>0$ such that if $M_{p / q}$ is the result of $p / q$ surgery on $K$ and $|p| \geq C \cdot g$, then every homomorphism from $\pi_{1}\left(M_{p / q}\right)$ to a torsion-free $n$-generator $\delta$-hyperbolic group is trivial.

Proof. By construction, if $a$ represents the core geodesic of $M_{p / q}$, we can estimate

$$
\operatorname{scl}(a) \leq g /|p| \text {. }
$$

Moreover, since $K$ is a knot in $S^{3}, \pi_{1}\left(M_{p / q}\right)$ is normally generated by $a$.

Let $G$ be $\delta$-hyperbolic and torsion free. Then no element is conjugate to its inverse, and therefore there is a uniform lower bound on the stable commutator length of any nontrivial element in $G$, depending only on $\delta$ and the size of a generating set for $G$. If $\rho: \pi_{1}\left(M_{p / q}\right) \rightarrow G$ is any homomorphism, then $\operatorname{scl}(\rho(a)) \leq \operatorname{scl}(a)$, and the image is normally generated by $\rho(a)$. The claim follows.

Remark 5.6. One knows that under a degree 1 map between hyperbolic manifolds of the same dimension, volume must go down. It is therefore significant in this corollary that the volumes of the manifolds $M_{p / q}$ go up as $|p| \rightarrow \infty$.

\section{First accumulation point}

In a torsion free word-hyperbolic group $G$, stable commutator length defines a function scl from conjugacy classes to $\mathbb{R}$. By Theorem $\mathrm{A}$ or $\mathrm{A}^{\prime}$, the first accumulation point $\delta_{\infty}$ for the image of this function is positive. In this section, we obtain universal estimates for $\delta_{\infty}$ which are independent of $G$. 
Theorem B (Accumulation Theorem). Let $G$ be a torsion free non-elementary wordhyperbolic group. Then the first accumulation point $\delta_{\infty}$ for stable commutator length satisfies

$$
\frac{1}{12} \leq \delta_{\infty} \leq \frac{1}{2}
$$

Proof. To obtain the upper bound, observe that $G$ contains a quasi-isometrically embedded copy of the free group on 2 generators. A nonabelian free group contains infinitely many conjugacy classes with $\mathrm{scl} \leq 1 / 2$ (see e.g. [1]). Since the embedding is quasi-isometric, the image of infinitely many of these conjugacy classes stay nonconjugate in $G$. Under any homomorphism, scl cannot go up, so the upper bound is proved.

An elementary argument gives a lower bound of $1 / 24$. We follow the argument in the proof of Theorem A, and we adopt notation and the setup from that theorem. In any hyperbolic group, there are only finitely many conjugacy classes of elements whose translation length is bounded above by any constant. Therefore, we may assume that the translation length of an element $a$ is as long as we like. In particular, we can assume that there is an axis $l_{a}$ which is geodesic and invariant under $a$. As before, let $\alpha$ denote a fundamental domain for $a^{n}$. Then length $(\alpha)=n \tau(a)$.

We suppose, as in the proof of Theorem A, that there is a segment $\sigma \subset l_{a}$ satisfying

$$
\text { length }(\sigma) \geq \frac{\text { length }(\alpha)-(4 m-2)\left(C_{3}^{\prime}+C_{4}^{\prime}\right)}{12 m-6}-2 C_{4}^{\prime},
$$

which cobounds a strip $R$ of $\widetilde{\Sigma}_{1}$ of width $\leq C_{5}$ with a translate $c\left(l_{a}\right)$. As before, there is $e=c a^{-1} c^{-1}$ which stabilizes $c\left(l_{a}\right)$ and moves points in almost the same direction as $a$.

If the translation length of $a$ is long enough, then under the assumption $\operatorname{scl}(a)<$ $1 / 24$, the estimate above gives us that length $(\sigma)$ is more than twice as big as a fundamental domain for $a$. For, if $\operatorname{scl}(a)<1 / 24$ then we can choose $n, m$ as above such that $n / m>24$. Now, length $(\alpha)=n \tau(a)$, so

$$
\text { length }(\sigma) \geq \frac{n}{12 m-6} \tau(a)-O(1) \geq 2 \tau(a),
$$

providing that $\tau(a)$ is sufficiently big.

In fact, we can assume that

$$
\frac{\text { length }(\sigma)}{2 \tau(a)} \geq 1+\epsilon
$$

where $\epsilon$ is any number smaller than $1 / 24-\operatorname{scl}(a)$. In particular, we can assume that

$$
\text { length }(\sigma)-2 \tau(a)=2 C_{6},
$$

where $C_{6}$ is as big as we like. We parameterize $\sigma$ as $\sigma(i)$ where

$$
i \in\left[\frac{-\mid \text { length }(\sigma) \mid}{2}, \frac{\mid \text { length }(\sigma) \mid}{2}\right] .
$$


Observe that for all $i \in\left[-C_{6}, C_{6}\right]$ we have estimates

$$
d\left(e^{-1} a(\sigma(i)), \sigma(i)\right) \leq 4 C_{5}, \quad d\left(a e^{-1}(\sigma(i)), \sigma(i)\right) \leq 4 C_{5} .
$$

For all $-C_{6} \leq i \leq C_{6}$ we let $\mu_{i} \in G$ be such that

$$
\mu_{i}(\sigma(0))=\sigma(i)
$$

It follows that for any $|i| \leq C_{6}$ we have

$$
d\left(\mu_{i}^{-1} e^{-1} a \mu_{i}(\sigma(0)), \sigma(0)\right) \leq 4 C_{5}, \quad d\left(\mu_{i}^{-1} a e^{-1} \mu_{i}(\sigma(0)), \sigma(0)\right) \leq 4 C_{5} .
$$

If $C_{6}$ is very big compared to $\left(|S|^{4 C_{5}}\right)^{2}$ then by the pigeonhole principle there are $i_{1}, \ldots, i_{n}$ where $n \geq C_{6} /\left(|S|^{4 C_{5}}\right)^{2}$ for which $\mu_{i_{j}} \mu_{i_{k}}^{-1}$ commutes with both $e^{-1} a$ and $a e^{-1}$ whenever $1 \leq j, k \leq n$. Up to this point, our argument makes no use of the fact that $G$ is torsion free.

In a torsion free word-hyperbolic group, two nontrivial elements which commute are proportional. Since $\mu_{i_{j}} \mu_{i_{k}}^{-1}$ commutes with both $e^{-1} a$ and $a e^{-1}$, it follows that $e^{-1} a$ and $a e^{-1}$ themselves are proportional. Since they are conjugate, they have the same translation length, and are either equal or inverse, since $G$ is torsion free. In the first case, $a$ and $e^{-1}$ commute; since they have the same (positive) translation length, they are either equal or inverse. Since their axes are almost oppositely aligned along $\sigma$, they must be inverse, so $a=e$. But $e=c a^{-1} c^{-1}$ which is absurd in a torsion free group. In the second case, $e^{-1} a=e a^{-1}$, so $e^{2}=a^{2}$, and therefore $e=a$ since $G$ is torsion free, and we get a contradiction in any case. This proves the estimate $\delta_{\infty} \geq 1 / 24$.

To get the estimate $\delta_{\infty} \geq 1 / 12$ we use Theorem 2.7. We argue as above that if $\operatorname{scl}(a)<1 / 12$ and $a$ has sufficiently long translation length, then

$$
\mid \text { length }(\sigma)-\tau(a) \mid=2 C_{6}
$$

is as big as we like. Let $p$ be a point at a distance $C_{6}$ from one of the endpoints of $\sigma$.

Let $p, q$ be the ideal points stabilized by $a$, and $r, s$ the ideal points stabilized by $b$. Then for suitable parameterizations of the flowlines $(p, q, \cdot),(r, s, \cdot)$ the points $(p, q, t)$ and $(r, s, t)$ are within distance $M e^{\lambda C_{6}}$ for $t \in\left[C_{6}\right.$, length $\left.(\sigma)-C_{6}\right]$, where $M$ and $\lambda<1$ are universal constants (depending only on $\delta$ and $|S|$ ), but $C_{6}$ is as big as we like.

This requires some explanation: by Theorem 2.7 (5), for any three ideal points $x$, $y, z$, there are parameterizations of $(x, z, \cdot)$ and $(y, z, \cdot)$ for which

$$
d^{\times}((x, z, t),(y, z, t)) \leq M \lambda^{t}
$$

for suitable $M$ and $0 \leq \lambda<1$. In fact, by Remark 2.9, these parameterizations are exactly those for which $(x, z, 0)$ is the closest point on $(x, z, \cdot)$ to $y$, and $(y, z, 0)$ is the closest point on $(y, z, \cdot)$ to $x$. Now, if $(p, q, \cdot)$ and $(r, s, \cdot)$ are flowlines 
which have long sub-segments which are distance $\leq C_{5}$ apart, then if $m$ is the point on $(p, q, \cdot)$ which is closest to $r$, and $n$ is the point on $(p, q, \cdot)$ which is closest to $s$, then $m, n$ are uniformly close to the endpoints of $\sigma$. Similarly, if $m^{\prime}, n^{\prime}$ are the points on $(r, s, \cdot)$ closest to $p, q$ respectively, then $m^{\prime}, m$ are close, and so are $n, n^{\prime}$. Now consider the flowline $(p, s, \cdot)$. This flowline converges exponentially fast to $(p, q, \cdot)$ along the ray from $n$ to $p$. Similarly, it converges exponentially fast to $(r, s, \cdot)$ along the ray from $m^{\prime}$ to $s$. Hence away from a bounded neighborhood of the endpoints of $\sigma$, the flowlines $(p, q, \cdot)$ and $(r, s, \cdot)$ are themselves exponentially close. This is the kind of convexity argument which is very standard in strictly negatively curved spaces; Mineyev's technology allows us to transplant it to $\mathcal{F}$.

It follows that

$$
d^{\times}\left(e^{-1} a \cdot\left(p, q, C_{6}\right),\left(p, q, C_{6}\right)\right) \leq 2 M e^{\lambda C_{6}} .
$$

By the triangle inequality, for any $n$ we estimate

$$
d^{\times}\left(\left(e^{-1} a\right)^{n} \cdot\left(p, q, C_{6}\right),\left(p, q, C_{6}\right)\right) \leq 2 n M e^{\lambda C_{6}}
$$

and therefore

$$
\tau\left(e^{-1} a\right) \leq \frac{2 n K M e^{\lambda C_{6}}+\epsilon}{n},
$$

where $K, \epsilon$ are as in the last bullet of Theorem 2.7. The constants $K, M, \lambda, \epsilon$ all depend only on $\delta$ and $|S|$, whereas we may choose $C_{6}$ as big as we like, and $n$ as big as we like. In particular, $\tau\left(e^{-1} a\right)$ may be made arbitrarily small by choosing $C_{6}$ very big.

On the other hand, if $a$ is not equal to $e$, then $\tau\left(e^{-1} a\right) \geq C_{1}>0$ for $C_{1}$ depending only on $\delta$ and $|S|$. This gives a contradiction for sufficiently big $C_{6}$ (chosen depending on $C_{1}$ ).

This contradiction proves the theorem.

Example 6.1. The upper bound $1 / 2$ is sharp, and is realized in a nonabelian free group, or closed hyperbolic surface group, by [7].

Example 6.2. Suppose that $H=\left\langle h_{1}, h_{2}\right\rangle$ is a non-free 2-generator subgroup of (any group) $G$. Then one can show $\operatorname{scl}\left(\left[h_{1}, h_{2}\right]\right)<1 / 2$. However, Delzant showed ([9]) that in any word-hyperbolic group there are only finitely many conjugacy classes of non-free 2-generator subgroups.

If $G$ is allowed to have torsion, things become slightly more complicated.

Example 6.3. Let $S$ be a (surface) orbifold containing two orbifold points $p_{2}, p_{3}$ of order 2 and 3 respectively. If $\alpha$ is any embedded arc in $S$ from $p_{2}$ to $p_{3}$, then the boundary of a regular neighborhood of $\alpha$ has scl at most 1/12. For typical $S$ there are infinitely many distinct isotopy classes of such $\operatorname{arcs} \alpha$. 
It is straightforward to see that in any word-hyperbolic group $G$, there is a positive first accumulation point for scl on conjugacy classes satisfying the no mirror condition (in fact this follows directly from Theorem A and Theorem $\mathrm{A}^{\prime}$ ), but we have not been able to show that this lower bound is independent of $G$, and therefore we pose the following

Question 6.4. Is there a universal positive constant $C$ such that the first accumulation point for scl on conjugacy classes satisfying the no mirror condition in a hyperbolic group is at least $C$ ?

Finally, the explicitness and universality of the constants in Theorem B, together with Example 6.1 motivates the following

Question 6.5. Can the lower bound in Theorem B be improved to $1 / 4$ ?

\section{Mapping class groups}

Our theorems may be generalized to groups which are not themselves hyperbolic but which act suitably on $\delta$-hyperbolic spaces. In this section and the next, we concentrate on two important examples.

We show how to adapt our Gap Theorem to the action of the mapping class group on the complex of curves. For an introduction to this complex and its properties, see [28] or [3]. The proof follows much the same outline as the proof of Theorem $\mathrm{A}^{\prime}$. A significant difference is that the action of $\operatorname{MCG}(S)$ on $\ell(S)$ is not proper; nevertheless it is weakly proper in a suitable sense [2], and this weak properness is enough. The technical tool we use is the acylindricity of the action of $\operatorname{MCG}(S)$ on $\ell(S)$ as observed by Bowditch [3].

We denote the translation length of an element $a \in \operatorname{MCG}(S)$ on $\mathcal{C}(S)$ by $\tau(a)$.

Theorem C (Mapping Class Theorem). Let $S$ be a compact orientable surface of hyperbolic type and $\mathrm{MCG}(S)$ its mapping class group. Then there is a positive integer $P$ depending on $S$ such that for any pseudo-Anosov element $a$, either there is an $0<n \leq P$ and an element $b \in \operatorname{MCG}(S)$ with $b a^{-n} b^{-1}=a^{n}$, or else there exists a homogeneous quasimorphism $h$ on $\operatorname{MCG}(S)$ such that $h(a)=1$ and $D(h) \leq C(S)$, where $C(S)$ depends only on $S$.

Moreover, let $a_{i} \in \operatorname{MCG}(S)$ be a collection of elements for which $T=\sup _{i} \tau\left(a_{i}\right)$ is finite. Suppose that for all integers $n, m \neq 0$ and all elements $b \in \operatorname{MCG}(S)$ and indices $i$ there is an inequality

$$
b a^{n} b^{-1} \neq a_{i}^{m}
$$

Then there is a homogeneous quasimorphism $h$ on $\operatorname{MCG}(S)$ such that

(1) $h(a)=1$, and $h\left(a_{i}\right)=0$ for all $i$,

(2) the defect of $h$ is $\leq C^{\prime}(S, T)$. 
Remark 7.1. By Thurston's classification of surface automorphisms (see e.g. [36]) every element of infinite order in $\operatorname{MCG}(S)$ is either reducible or pseudo-Anosov. An element $a \in \operatorname{MCG}(S)$ has $\tau(a)=0$ on $\ell(S)$ if and only if it has finite order or it is reducible.

Remark 7.2. Note the reappearance of the no mirror condition. Also note the separation theorem; compare with the statement of Theorem $\mathrm{A}^{\prime}$.

Remark 7.3. The dependence of $C(S)$ on $S$ is somewhat subtle and indirect, and does not seem to be easy to estimate.

Proof. The basic structure of the proof should be reasonably clear at this point.

Let $\ell(S)$ be the curve graph of $S . \digamma(S)$ is $\delta$-hyperbolic [28]. Any pseudoAnosov element acts as an axial isometry. Moreover, by Bowditch [3], there exists $B(S)$ such that for any pseudo-Anosov element $a, a^{B}$ has a geodesic axis in $\ell(S)$. So, in particular, $\tau(a) \geq 1 / B$.

If there exists $n>0$ and $b \in \operatorname{MCG}(S)$ with $b a^{n} b^{-1}=a^{-n}$, then one may assume that $n \leq P(S)$, where $P(S)$ depends only on $S$. This follows because the action of $\operatorname{MCG}(S)$ on $\mathcal{C}(S)$ is acylindrical in the sense of Bowditch [3]. Here is the precise statement of acylindricity: for any $A>0$, there exists $E, M$ such that for any two points $x, y \in \mathcal{C}(S)$ with $d(x, y) \geq E$ then there are at most $M$ elements $b \in \operatorname{MCG}(S)$ such that $d(x, b x) \leq A, d(y, b y) \leq A$. We consider the case $A=10 \delta$. So, in the following we assume that for all $0<n$ and $b \in \operatorname{MCG}(S)$, we have $b a^{n} b^{-1} \neq a^{-n}$.

From above, one finds that there is $P^{\prime}>0$ such that if $n \geq P^{\prime}$, then (a copy of the geodesic segment) $a^{-n}$ does not appear in the $L$-neighborhood of a geodesic axis of $a^{B}$, where $L$ is the constant from Section 4 , which depends only on $\delta$ in this setting. This is because, otherwise, one finds that $a^{m}$ is conjugate to $a^{-m}$ for some $0<m$, which is a contradiction.

So there exists $R(S)$ such that for any $a$ as in the theorem there exists $N \leq R$ such that $a^{N}$ has a geodesic axis, and no reverse counting happens for $a^{N}$.

Let $\alpha$ be a geodesic axis for $a^{N}$. Let $x \in \alpha$, and denote the subpath from $x$ to $a^{N} x$ as $a^{N}$. Let $h=c-c_{-}$be the quasimorphism constructed using the counting functions for the pairs $\left(a^{N}, 1\right)$ and $\left(a^{-N}, 1\right)$. Then $c\left(a^{N n}\right) \geq n$ and $c_{-}\left(a^{N n}\right)=0$ for any $n>0$, since there is no reverse counting. So, $h\left(a^{N n}\right) \geq n$ for all $n>0$. We know that $D(h) \leq K(\delta)$, where $K(\delta)$ depends only on $S$ by Proposition 4.4. Therefore, given $a$, we can construct (by averaging $h$ ) a homogeneous quasimorphism $f$ such that $f(a)=1$ and $D(f) \leq 4 K R$, where the constant $K R$ depends only on $S$.

The argument to prove the second part is very similar to the proof of the second part of Theorem A'. Given a collection of elements $a_{i}$ and a uniform upper bound $T$ on their translation lengths, if there is a translate of the axis of $a$ which stays close to an axis of $a_{i}$ on a sufficiently long interval, then by acylindricity and the pigeonhole principle, we can conclude that some power of $a$ is conjugate to a power of $a_{i}$. 
In slightly more detail: let $l_{a_{i}}$ be an axis for $a_{i}$, and let $v$ be an axis for a conjugate $e=c a c^{-1}$ of $a$ which stays close to $l_{a_{i}}$ on a sufficiently long segment $\beta$ (we say how long in a moment). Let integers $n_{j}, m_{j}$ be chosen for which

$$
\left|n_{j} \tau\left(a_{i}\right)-m_{j} \tau(a)\right|<C_{1}
$$

for some fixed constant $C_{1}$. Given an upper bound on $T$ and $\tau(a)$, we can find at least $C_{2}$ many such pairs $\left(n_{j}, m_{j}\right)$ whose absolute values are bounded by $C_{3}$ where $C_{2}$ is as big as we like, and where $C_{3}$ depends on $T, \tau(a), S, C_{1}$ (and not on the specific element $a_{i}$ ). Let $\beta$ be longer than $C_{3} T, C_{3} \tau(a)$. Then (after possibly replacing $n_{j}$ by $-n_{j}$ for some $j$ ) we observe that

$$
d\left(a_{i}^{n_{j}} e^{m_{j}}(p), p\right) \leq C_{1}^{\prime}
$$

for some $C_{1}^{\prime}$ which depends only on $C_{1}$ and $S$. By acylindricity and the pigeonhole principle, there are distinct indices (which we denote by $n_{1}, m_{1}, n_{2}, m_{2}$, respectively) for which

$$
a_{i}^{n_{1}} e^{m_{1}}=a_{i}^{n_{2}} e^{m_{2}}
$$

and therefore some nonzero power of $a_{i}$ is equal to some nonzero power of $e$, which is itself conjugate to $a$, contrary to hypothesis.

This shows that the length $\beta$ can be bounded above in terms of $S, T, \tau(a)$.

Note that one constructs by this argument a single quasimorphism $h$ whose value grows linearly on powers of $a$, and which vanishes on all powers of $a_{i}$ simultaneously. The fact that there are (typically) infinitely many $a_{i}$ on which $h$ vanishes is immaterial.

We give another proof of the second claim which makes more explicit use of acylindricity and gives the slightly better constants claimed in the statement of the theorem.

The argument is similar to the one to show for sufficiently large $N$, there is no reverse counting for $a^{N}$, since otherwise, for some $0<n \leq N, a^{n}$ is conjugate to $a^{-n}$ by the acylindricity of the action, which is impossible. Also, see the proof of Claim $2^{\prime}$ in the proof of Theorem $\mathrm{A}^{\prime}$.

We want to show that $c_{a^{ \pm 2 N}}\left(a_{i}\right)=0$ for all $i$ if $N$ is bigger than a constant depending only on $S$ and $T$. Suppose that $c_{a^{2 N}}\left(a_{i}\right)>0$ for some (fixed) $i$ (the argument is precisely analogous for $\left.c_{a^{-2 N}}\right)$. Let $l_{a_{i}}$ be an axis for $a_{i}$, and let $l_{e}$ be an axis for a conjugate $e=c a c^{-1}$ of $a$ which stays close to, namely in the $L(\delta)$ neighborhood of, $l_{a_{i}}$ on a segment $v$ such that one endpoint is $v_{0}$ and the other end point is $e^{2 N}\left(v_{0}\right)$.

For simplicity, we assume that both $l_{a_{i}}, l_{e}$ are geodesics (replace $a, a_{i}$ by $a^{B}$, $a_{i}^{B}$ if necessary). Then $v$ is $2 \delta$-close to $l_{a_{i}}$, where $\delta$ is the hyperbolicity constant for $\mathcal{C}(S)$ (here we assume that $v$ is much longer than $\delta$, which follows if we take $N$ bigger than a constant depending only on $S$ ).

We observe that for all $0 \leq j \leq N$, there exists $n_{j}$ such that

$$
d\left(a_{i}^{n_{j}} e^{j}\left(v_{0}\right), v_{0}\right), d\left(a_{i}^{n_{j}} e^{j+N}\left(v_{0}\right), e^{N}\left(v_{0}\right)\right) \leq \tau\left(a_{i}\right)+4 \delta \leq T+4 \delta .
$$


We set $A=T+4 \delta$ and obtain corresponding constants $D(A), M(A)$ for the acylindricity of the action. Now assume that $N$ is such that $d\left(e^{N}\left(v_{0}\right), v_{0}\right) \geq D(A)$. In other words, $N \tau(a) \geq D(A)$ (we know $\tau(a) \geq 1 / B$, so take $N \geq B D$ ). Then, by the acylindricity, there are at most $M$ distinct elements in $a_{i}^{n_{j}} e^{j}(0 \leq j \leq N)$. It follows that if $N>M$, then some non-trivial power of $a$ is conjugated by $c$ to a (possibly trivial) power of $a_{i}$, which is impossible. We thereby obtain an upper bound for $N$ by $A, D(A), M(A), B$, which depend only on $S, T$, to have $c_{a^{2 N}}\left(a_{i}\right)>0$. We obtain the same upper bound from $c_{a^{-2 N}}\left(a_{i}\right)>0$ as well.

\section{Amalgamations}

In this section we adapt our theorem to the special case of an amalgamated free product acting on its associated tree, and construct many quasimorphisms with uniform lower bounds. One must be slightly careful: the group $\operatorname{SL}\left(2, \mathbb{Q}_{p}\right)$ is an amalgam of two copies of $\operatorname{SL}\left(2, \mathbb{Z}_{p}\right)$. Nevertheless, $\operatorname{SL}\left(2, \mathbb{Q}_{p}\right)$ is uniformly perfect, and therefore admits no nonzero homogeneous quasimorphisms at all. As in the case of the mapping class group acting on $\mathcal{C}(S)$, one must ensure (by fiat) that the action of the amalgam on its associated tree is weakly proper; this is guaranteed by a suitable malnormality condition.

Theorem D (Amalgamation Theorem). Let $G=A *_{C} B$. Let $w$ be a word on $A, B$ which is reduced and cyclically reduced such that $|w|>1$, and let $\bar{w}$ denote the corresponding element in $G$. Suppose that the double coset $C \bar{w} C$ does not contain the element corresponding to any cyclic conjugate of $w^{-1}$. Then there exists a homogeneous quasimorphism $h$ on $G$ such that $h(\bar{w})=1$, and the defect of $h$ is $\leq 312$.

Proof. We use [17]. Here is a review. Let $\Gamma$ be the Cayley graph of $G$ for the generating set $A \cup B$.

Then a geodesic between 1 and $a$ is exactly a word for $a$ reduced as a word in $A *_{C} B$ (Lemma 3.1 [17]). Let $w$ be a reduced word for $a$. Assume that $w$ is cyclically reduced. Then $w^{*}=\ldots w w w \ldots$ is an infinite reduced word, which is a geodesic in $\Gamma$ since $w$ is cyclically reduced. For such $w$, let $c, c_{-1}$ be the counting function for $(w, 1),\left(w^{-1}, 1\right)$. Then $c\left(a^{n}\right) \geq n$ for all $n>0$.

By the double coset condition, $c_{-1}\left(a^{n}\right)=0$ for all $n>0$, namely, no reverse counting. The argument is essentially in [17]. Suppose that $c_{-1}\left(a^{n}\right)>0$. Then there is a realizing path $\alpha$ from 1 to $a^{n}$ which contains $w^{-1}$ as a subword. It is shown in Lemma 3.2 [17] that one can always take a realizing path to be a geodesic. Let $\beta=w^{n}$, which is a geodesic from 1 to $a^{n}$. Since both reduced words $\alpha$ and $\beta$ represent the same element $a^{n}$, by Britton's lemma, there must be $c, c^{\prime} \in C$ such that $c \bar{w} c^{\prime}=\bar{v}$, where $v$ is some cyclic conjugate of $w^{-1}$ (see Section 4 of [17] for details). But this is prohibited. 
It follows that $h\left(a^{n}\right) \geq n$, where $h=c-c_{-1}$. It is shown in Proposition 3.1 [17] that $D(h) \leq 78$. When we make a quasimorphism homogeneous, an upper bound for the defect becomes $312=78 \times 4$.

Remark 8.1. There is no implied suggestion that the constant 312 is optimal! The main point is that it is a universal constant, which does not depend in any way on the group $G$.

Remark 8.2. If $C$ is trivial, so that $G$ is a free product, Bavard ([1], Proposition 3.7.2) obtains the (optimal) lower bound $\mathrm{scl} \geq 1 / 2$.

Remark 8.3. Note that the double coset condition is the precise analogue of the no mirror condition in the context of amalgamated free products.

Question 8.4. What is the optimal constant in Theorem D?

The following example clarifies the geometry of the double coset condition in the context of 3-manifold topology.

Example 8.5. Let $M$ be a knot complement in a rational homology sphere. After choosing an orientation for the knot and for $M$, there is a natural choice of meridian and longitude $m, l$ on $\partial M$. The longitude is defined by the property that it is virtually trivial in homology. So there is some surface $S$ of least Euler characteristic in $M$ whose boundary is a multiple of the longitude.

Suppose that $[\partial S]=n \cdot[l]$ in homology. Define $\chi_{\mathbb{Q}}=\frac{\chi(S)}{n}$. Let $M_{p / q}$ be the result of Dehn filling $M$ along the slope $p / q$. Let $\gamma \subset M_{p / q}$ denote the core of the added solid torus. As in Example 2.3, we have an estimate for stable commutator length

$$
\operatorname{scl}(\gamma) \leq \frac{-\chi \mathbb{Q}}{2 p}
$$

As before, for $K$ a knot in $S^{3}$, this estimate becomes

$$
\operatorname{scl}(\gamma) \leq \frac{g-1 / 2}{p},
$$

where $g$ is the genus of a Seifert surface for $K$.

A slope $p / q$ on $\partial M$ is said to be a boundary slope if there is an essential, oriented, proper surface $\Sigma \subset M$ such that $\partial \Sigma$ is a nonempty union of curves isotopic to the $p / q$ curve, with either orientation.

After filling $M$ to $M_{p / q}$, the manifold $M_{p / q}$ splits along a surface $\Sigma^{\prime}$ obtained by filling in the boundary components of $\Sigma$, into two submanifolds which by abuse of notation we denote $M_{A}, M_{B}$ with fundamental groups $A, B$. For brevity, we denote $\pi_{1}(\Sigma)=C$. The core geodesic $\gamma$ intersects the two submanifolds $M_{A}, M_{B}$ efficiently, in a collection of proper arcs which represent elements of the double coset 
spaces $C A C$ and $C B C$. If the volume of $M$ (and therefore that of $M_{p / q}$ ) is small, and the area of $\Sigma^{\prime}$ is large, most of $M_{A}, M_{B}$ must be very thin, and have the structure of an $I$-bundle over some subsurfaces $\Sigma_{A}^{\prime}, \Sigma_{B}^{\prime}$ of $\Sigma^{\prime}=\partial M_{A}=\partial M_{B}$. These $I$-bundles are known as the characteristic submanifolds of $M_{A}, M_{B}$ and we denote them by $I_{A}$, $I_{B}$, respectively.

If $\Sigma^{\prime}$ is connected, the boundary components of $I_{A}$ and $I_{B}$ are contained in a single surface. Suppose that $\partial I_{A}$ and $\partial I_{B}$ are connected. The geodesic $\gamma$ is decomposed into a sequence of geodesic segments $\gamma_{j}$ with $0 \leq j \leq i$ which are the connected components of $\gamma \cap\left(M_{p / q}-\Sigma^{\prime}\right)$. Each $\gamma_{i}$ is properly embedded in $M_{A}$ or $M_{B}$, and is contained in the corresponding characteristic submanifold $I_{A}$ or $I_{B}$. Since we are assuming $\partial I_{A}, \partial I_{B}$ are connected, each oriented geodesic segment $\gamma_{j}$ can be dragged around $I_{A}$ (say) into itself in such a way that the orientation is reversed at the end. Composing these proper isotopies, we can drag all the $\gamma_{j}$ simultaneously in such a way that the common endpoints of $\gamma_{j}$ and $\gamma_{j-1}$ agree during the dragging, for each $j \leq i$. The initial point of $\gamma_{0}$ and the final point of $\gamma_{i}$ will not necessarily follow homotopic paths under this dragging, and their difference is an element of $\pi_{1}\left(\Sigma^{\prime}\right)$. Translating this into algebraic information, we have exhibited a conjugate of $\gamma$ as an element of the double coset space $\pi_{1}\left(\Sigma^{\prime}\right) \gamma^{-1} \pi_{1}\left(\Sigma^{\prime}\right)$.

This example actually occurs: Nathan Dunfield [11] has produced examples of Montesinos knots with genus 1 for which $p / 1$ is an essential slope where $p \sim$ 20,000. The corresponding core geodesics $\gamma \operatorname{satisfy} \operatorname{scl}(\gamma) \leq \frac{1}{40,000}$ and therefore $\gamma$ is conjugate into $\pi_{1}\left(\Sigma^{\prime}\right) \gamma^{-1} \pi_{1}\left(\Sigma^{\prime}\right)$ by Theorem $\mathrm{D}$.

The double coset condition shows us how to think about the geometry of the resulting manifolds, and the way in which $\gamma$ sits inside them.

\section{References}

[1] C. Bavard, Longueur stable des commutateurs. Enseign. Math. (2) 37 (1991), 109-150. Zbl 0810.20026 MR 1115747

[2] M. Bestvina and K. Fujiwara, Bounded cohomology of subgroups of mapping class groups. Geom. Topol. 6 (2002), 69-89. Zbl 1021.57001 MR 1914565

[3] B. H. Bowditch, Tight geodesics in the curve complex. Invent. Math. 171 (2008), 281-300. Zbl 05248088 MR 2367021

[4] R. Brooks, Some remarks on bounded cohomology. In Riemann surfaces and related topics: Proceedings of the 1978 Stony Brook Conference, Ann. of Math. Stud. 97, Princeton University Press, Princeton, N.J., 1981, 53-63. Zbl 0457.55002 MR 0624804

[5] D. Calegari, Length and stable length. Geom. Funct. Anal. 18 (2008), 50-76. Zbl 1155.57015 MR 2399095

[6] D. Calegari, scl. MSJ Memoirs 20, Math. Soc. Japan, Tokyo 2009. MR 2527432

[7] L. P. Comerford, Jr. and C. C. Edmunds, Products of commutators and products of squares in a free group. Internat. J. Algebra Comput. 4 (1994), 469-480. Zbl 0808.20027 MR 1297152 
[8] M. Culler, Using surfaces to solve equations in free groups. Topology 20 (1981), 133-145. Zbl 0452.20038 MR 605653

[9] T. Delzant, Sous-groupes à deux générateurs des groupes hyperboliques. In Group theory from a geometrical viewpoint (Trieste, 1990), World Sci. Publ., Singapore 1991, 177-189. Zbl 0845.20027 MR 1170366

[10] A. J. Duncan and J. Howie, The genus problem for one-relator products of locally indicable groups. Math. Z. 208 (1991), 225-237. Zbl 0724.20024 MR 1128707

[11] N. Dunfield, private communication.

[12] H. Endo and D. Kotschick, Bounded cohomology and non-uniform perfection of mapping class groups. Invent. Math. 144 (2001), 169-175. Zbl 0987.57004 MR 1821147

[13] H. Endo and D. Kotschick, Failure of separation by quasi-homomorphisms in mapping class groups. Proc. Amer. Math. Soc. 135 (2007), 2747-2750. Zbl 1127.20029 MR 2317948

[14] D. B. A. Epstein and K. Fujiwara, The second bounded cohomology of word-hyperbolic groups. Topology 36 (1997), 1275-1289. Zbl 0884.55005 MR 1452851

[15] B. Farb, C. J. Leininger, and D. Margalit, The lower central series and pseudo-Anosov dilatations. Amer. J. Math. 130 (2008), 799-827. Zbl 05300184 MR 2418928

[16] K. Fujiwara, The second bounded cohomology of a group acting on a Gromov-hyperbolic space. Proc. London Math. Soc. (3) 76 (1998), 70-94. Zbl 0891.20027 MR 1476898

[17] K. Fujiwara, The second bounded cohomology of an amalgamated free product of groups. Trans. Amer. Math. Soc. 352 (2000), 1113-1129. Zbl 1053.20517 MR 1491864

[18] S. M. Gersten, Cohomological lower bounds for isoperimetric functions on groups. Topology 37 (1998), 1031-1072. Zbl 0933.20026 MR 1650363

[19] É. Ghys and V. Sergiescu, Sur un groupe remarquable de difféomorphismes du cercle. Comment. Math. Helv. 62 (1987), 185-239. Zbl 0647.58009 MR 896095

[20] M. Gromov, Volume and bounded cohomology. Inst. Hautes Études Sci. Publ. Math. 56 (1982), 5-99. Zbl 0516.53046 MR 686042

[21] M. Gromov, Hyperbolic groups. In Essays in group theory, Math. Sci. Res. Inst. Publ. 8, Springer-Verlag, New York 1987, 75-263. Zbl 0634.20015 MR 0919829

[22] M. Gromov, Geometric group theory (Sussex, 1991), vol. 2: Asymptotic invariants of infinite groups. London Math. Soc. Lecture Note Ser. 182, Cambridge University Press, Cambridge 1993. Zbl 0841.20039 MR 1253544

[23] D. Groves and J. F. Manning, Dehn filling in relatively hyperbolic groups. Israel J. Math. 168 (2008), 317-429. Zbl 05508729 MR 2448064

[24] U. Hamenstädt, Bounded cohomology and isometry groups of hyperbolic spaces. J. Eur. Math. Soc. (JEMS) 10 (2008), 315-349. Zbl 1139.22006 MR 2390326

[25] D. Kotschick, Signatures, monopoles and mapping class groups. Math. Res. Lett. 5 (1998), 227-234. Zbl 0905.57010 MR 1617905

[26] D. Kotschick, Quasi-homomorphisms and stable lengths in mapping class groups. Proc. Amer. Math. Soc. 132 (2004), 3167-3175. Zbl 1055.20037 MR 2073290 
[27] D. Kotschick, Stable length in stable groups. In Groups of diffeomorphisms: in honor of Shigeyuki Morita on the occasion of his 60th birthday Adv. Stud. Pure Math. 52, Kinokuniya, Tokyo 2008, 401-414.

[28] H. A. Masur and Y. N. Minsky, Geometry of the complex of curves I: Hyperbolicity. Invent. Math. 138 (1999), 103-149. Zbl 0941.32012 MR 1714338

[29] I. Mineyev, Flows and joins of metric spaces. Geom. Topol. 9 (2005), 403-482. Zbl 1137.37314 MR 2140987

[30] I. Mineyev, N. Monod, and Y. Shalom, Ideal bicombings for hyperbolic groups and applications. Topology 43 (2004), 1319-1344. Zbl 1137.20033 MR 2081428

[31] D. V. Osin, Peripheral fillings of relatively hyperbolic groups. Invent. Math. 167 (2007), 295-326. Zbl 1116.20031 MR 2270456

[32] J.-C. Picaud, Cohomologie bornée des surfaces et courants géodésiques. Bull. Soc. Math. France 125 (1997), 115-142. Zbl 0888.53049 MR 1459300

[33] L. Polterovich, Growth of maps, distortion in groups and symplectic geometry. Invent. Math. 150 (2002), 655-686. Zbl 1036.53064 MR 1946555

[34] L. Polterovich and Z. Rudnick, Stable mixing for cat maps and quasi-morphisms of the modular group. Ergodic Theory Dynam. Systems 24 (2004), 609-619. Zbl 1071.37019 MR 2054053

[35] W. Thurston, The geometry and topology of three-manifolds. Princeton University, Princeton 1980. http://www.msri.org/publications/books/gt3m/

[36] W. P. Thurston, On the geometry and dynamics of diffeomorphisms of surfaces. Bull. Amer. Math. Soc. (N.S.) 19 (1988), 417-431. Zbl 0674.57008 MR 956596

Received June 6, 2008; revised June 22, 2009

D. Calegari, Department of Mathematics, California Institute of Technology, Pasadena CA, 91125, U.S.A.

E-mail: dannyc@its.caltech.edu

K. Fujiwara, Graduate School of Information Science, Tohoku University, Sendai, Japan

E-mail: fujiwara@math.is.tohoku.ac.jp 